\title{
A Comparative Study on the Performance of LLR- and SNR-Based Hybrid Relaying Schemes
}

\author{
Ahmed El-Mahdy ${ }^{1}$ and Wassim Alexan ${ }^{2}$ \\ ${ }^{1}$ Faculty of Information Engineering and Technology, The German University in Cairo, Cairo, Egypt \\ ${ }^{2}$ The German University in Cairo, Cairo, Egypt \\ Correspondence should be addressed to Wassim Alexan; wassim.joseph@guc.edu.eg
}

Received 23 July 2016; Accepted 7 September 2016; Published 10 January 2017

Academic Editor: Pierre-Martin Tardif

Copyright (c) 2017 A. El-Mahdy and W. Alexan. This is an open access article distributed under the Creative Commons Attribution License, which permits unrestricted use, distribution, and reproduction in any medium, provided the original work is properly cited.

A comparative study on the theoretical bit error rate (BER) is presented for hybrid relaying schemes that toggle between adaptive decode-and-forward (ADF) and amplify-and-forward (AF) protocols, for a typical three-node wireless network. Toggling between the two forwarding protocols is based on the log-likelihood ratio (LLR) or the signal-to-noise ratio (SNR) of the received signal at the relay node. Closed-form expressions for the probability of error are presented, as well as the expressions of the proposed schemes' gains over classical ADF and AF protocols. Comparisons are carried out among the two schemes and other hybrid schemes found in the literature. Moreover, the impact of relay location on the probability of error is investigated.

\section{Introduction}

Since the early works of [1-5] on cooperative communications, the wireless communications community has come to realize the tremendous advantages that could be attained through the utilization of relay nodes in a wireless network, namely, gains in spatial diversity, improved cellular coverage, and the potential of increased capacity.

Relaying schemes are generally classified according to the forwarding protocol employed at the relay nodes. The two most common forwarding protocols are amplify-andforward (AF) and decode-and-forward (DF). A relay node employing an AF protocol simply amplifies the received signal from the source node and forwards it to the destination node. No signal processing whatsoever is carried out at the relay node, thus significantly saving up on the cooperation overhead and reducing system complexity. Nevertheless, this forwarding protocol has the drawback of amplifying the noise as well. On the other hand, a relay node employing a DF protocol would decode the received signal from the source node, before encoding it once again and forwarding it to the destination node. Moreover, DF protocol is subclassified into (a) fixed decode-and-forward (FDF) protocol, whereby the relay node always forwards the received signal, thus allowing for errors occurring in the $S \rightarrow R$ link to propagate through the $R \rightarrow D$ link, ultimately leading to wrong decisions at $D$, and (b) adaptive decode-and-forward (ADF) protocol, whereby the received signal from $S$ is only forwarded by $R$ under the condition of probable correct decoding. Such a condition is usually checked through the satisfaction of a certain metric, for example, if the measured SNR is higher than a certain predefined threshold, denoted by $\eta_{\mathrm{SNR}}$.

Hybrid relaying schemes that switch between AF and FDF protocols were tackled in [6-8]. In $[6,7]$, the authors provide simulations for a relaying system that allows $R$ to toggle between FDF, AF, and no-send protocols by comparing either the measured SNR or the calculated LLR of the received signal at $R$ with predefined thresholds. In [8], a closed-form expression is provided for the proposed relaying scheme, which allows $R$ to perform soft decoding and then forwards the reliability information at the output of its decoder to $D$.

Hybrid relaying schemes that switch between AF and $\mathrm{ADF}$ protocols were tackled in [9-11]. In [9], a closedform expression of the symbol-error-rate (SER), for M-PSKmodulated signals, is provided for the hybrid scheme. In their hybrid scheme, the authors allow $R$ to toggle between ADF and AF protocols based on the mathematical calculation of the probability of the relay's ability to correctly decode the 
received signal from $S$. In reality, this is not very practical, so the authors of [10] choose to allow $R$ to base its decision on the SNR of the received signal. In their proposed scheme, $R$ employs DF protocol in case the SNR of the received signal exceeds $\eta_{\mathrm{SNR}}$; otherwise it employs AF. A closed-form expression of the BER, only for BPSK-modulated signals, is provided in their work. The authors of [11] clarify that, for SNR-based hybrid relaying, it would be better for $R$ to employ AF protocol if the SNR of the received signal from $S$ exceeds $\eta_{\mathrm{SNR}}$, otherwise $R$ would employ ADF protocol. This is because $S \rightarrow R$ link is already in a "good state." A closedform expression of the SER for MPSK-modulated signals is provided in their work.

In this paper, analysis of the performance of a hybrid relaying protocol that toggles between $\mathrm{ADF}$ and AF protocols, according to the quality of the received signal from $S$ at $R$, is proposed. A comparative study between different metrics, LLR and SNR, is carried out. The contributions of this paper can be summarized in the following points: (a) a hybrid relaying protocol that employs either ADF or AF protocol, based on the LLR of the received signal from $S$ at $R$, is proposed and (b) a hybrid relaying protocol that employs either ADF or AF protocol, based on the SNR of the received signal from $S$ at $R$, is proposed. Note that the proposed approach distinguishes itself from other SNR-based approaches in the literature by employing ADF protocol instead of DF protocol. (c) The proposed protocols are analyzed by deriving the closed-form expressions for the BER BPSK-modulated signals. (d) The performance of the proposed protocols is compared with that of other hybrid protocols. (e) The proposed protocols' performance is demonstrated to outperform their counterparts, through several examples. (f) The effect of the $S$ - $R$ separation on the performance of the proposed protocols is analyzed. $(\mathrm{g})$ The relaying gain of the proposed protocols over ADF and AF protocols is investigated. (h) For the high SNR regime, it is shown that the performance of the proposed protocols is identical to that of ADF protocol.

The rest of this paper is organized as follows. In Section 2, the system model is described. In Section 3, the proposed LLR- and SNR-based hybrid relaying schemes are outlined and the mathematics of the LLR calculation is laid out. In Section 4, the performance of the proposed schemes is analyzed by deriving closed-form expressions for the BER, and the performance gain over the ADF and AF relaying protocols is evaluated. In Section 5, numerical results are presented and interpreted. Comparisons to other hybrid relaying schemes, found in the literature, are also carried out. Finally, the conclusions are drawn in Section 6.

Throughout this paper, the following definitions and notations will be used. The source, relay, and destination nodes are denoted, respectively, by $S, R$, and $D$. Furthermore, the superscripts $(\cdot)^{*}$ and $(\cdot)^{C}$ denote the complex conjugate and the complementary event; $\operatorname{Re}[\cdot]$ and $E[\cdot]$ denote the real part and the statistical expectation, respectively.

\section{System Model}

A cooperative system comprising a single $S$, a single $R$, and a single $D$, as shown in Figure 1, is considered in this paper,

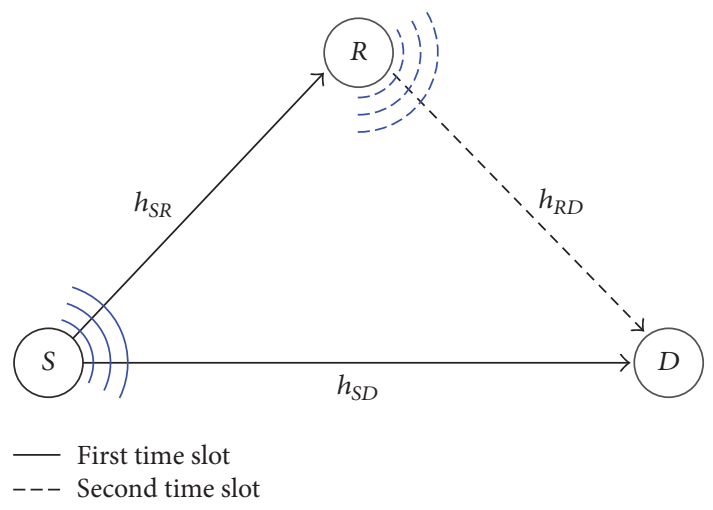

Figure 1: A simplified cooperative communication system model.

where the direct communication is assisted by $R$. To avoid interference from simultaneous transmissions, $R$ is assumed to operate in the half duplex mode and thus each transmission consists of two time slots, where each time slot spans a single frame interval. In the first time slot, $S$ broadcasts its message signal, which is received by $R$ and $D$. In the second time slot, if $R$ employs ADF protocol, the received message from $S$ is decoded and forwarded to $D$; otherwise if $R$ employs AF protocol, the received message from $S$ is forwarded with an amplifying factor $G$. In order to allow for equal power allocation between $S$ and $R$, the choice for $G$ is varied accordingly with each time slot. As in [9] and for a simpler analysis, orthogonal $\mathrm{AF}(\mathrm{OAF})$ protocol is considered (i.e., $S$ remains silent during the second slot). Finally, $D$ combines the transmissions from the two time slots, utilizing the maximal-ratio-combining (MRC) technique, thus maximizing the reliability. Furthermore, quasi-static fading is assumed, such that, for the duration of a single frame, the channel is assumed to be constant and only to change value independently for each subsequent new frame. During the first time slot, the received signals from $S$ at $R$ and $D$ are given, respectively, by

$$
\begin{aligned}
& y_{S R}=h_{S R} x+n_{S R}, \\
& y_{S D}=h_{S D} x+n_{S D},
\end{aligned}
$$

where $x$ is the transmitted symbol from S. $x$ is assumed to have an average energy per bit $E_{b}$ and to belong to the constellation set of BPSK. During the second time slot and based on the employed relaying mode, whether ADF or AF, the received signals at $D$ from $R$ are given by

$$
\begin{aligned}
y_{R D}^{\mathrm{ADF}} & =h_{R D} x+n_{R D}, \\
y_{R D}^{\mathrm{AF}} & =h_{R D} G y_{S R}+n_{R D} .
\end{aligned}
$$

In (1) and (2), the fading channel magnitudes, received signals, and additive white Gaussian noise (AWGN) with variance $N_{0}$ are, respectively, denoted by $h_{A}, y_{A}$, and $n_{A}$ with $A \in\{S D, S R, R D\}$ and channel mean power $\Omega_{A}$, where 
$h_{A} \sim \mathscr{C} \mathscr{N}\left(0, \Omega_{A}\right)$ denotes the complex Gaussian random variable with zero-mean and variance $\Omega_{A}$ and $n_{A} \sim$ $\mathscr{C} \mathscr{N}\left(0, N_{0}\right)$ are statistically independent. For the AF mode, the amplifying factor $G$ is chosen as [6]

$$
G=\frac{1}{\sqrt{\left|h_{S R}\right|^{2}+1 / \gamma_{0}}},
$$

where $\gamma_{0}$ is the SNR of the received signal at $R$.

\section{Proposed HDAF Relaying Schemes and LLR Calculation}

For the LLR-based HDAF and for each frame, the LLR value at $R, \Lambda_{S R}$ is calculated and compared to a predetermined threshold, $\eta_{\text {LLR }}$. If $\Lambda_{S R}$ exceeds $\eta_{\text {LLR }}, R$ employs the ADF protocol; otherwise, it employs the AF protocol. That is,

$$
\Lambda_{S R} \underset{\mathrm{AF}}{\stackrel{\mathrm{ADF}}{\gtrless}} \eta_{\mathrm{LLR}} \cdot
$$

For BPSK, $\Lambda_{S R}$ is calculated as [12]

$$
\begin{aligned}
\Lambda_{S R} & =\ln \left[\frac{P\left\{x_{v}|| h_{S R} \mid, y_{S R}\right\}}{P\left\{x_{w}|| h_{S R} \mid, y_{S R}\right\}}\right] \\
& =\ln \left[\frac{\operatorname{Exp}\left[-\left.\left.\left|y_{S R}-\right| h_{S R}\right|^{2} \sqrt{E_{b}}\right|^{2} /\left|h_{S R}\right|^{2} \sigma_{S R}^{2}\right]}{\operatorname{Exp}\left[-\left.\left.\left|y_{S R}+\right| h_{S R}\right|^{2} \sqrt{E_{b}}\right|^{2} /\left|h_{S R}\right|^{2} \sigma_{S R}^{2}\right]}\right] \\
& =\frac{4 \sqrt{E_{b}}}{\sigma_{S R}^{2}} \operatorname{Re}\left\{y_{S R}\right\},
\end{aligned}
$$

where $x_{v}$ and $x_{w}$ are any two different symbols from the BPSK constellation set, $\left\{+\sqrt{E_{b}},-\sqrt{E_{b}}\right\}$.

For the SNR-based HDAF and for each frame, the measured $S N R$ value at $R, \Gamma_{S R}$ is compared to a predetermined threshold, $\eta_{\mathrm{SNR}}$. If $\Gamma_{S R}$ exceeds $\eta_{\mathrm{SNR}}, R$ employs the ADF protocol; otherwise, it employs the AF protocol. That is,

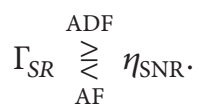

For BPSK, $\Gamma_{S R}$ is calculated as

$$
\Gamma_{S R}=\frac{\left(d_{S R}\right)^{-a}}{\sigma_{S R}^{2}} E_{b}
$$

where $d_{S R}$ is the normalized distance between $S$ and $R$, while $a$ is the path-loss exponent.

\section{Bit Error Probability and Relaying Gain}

4.1. Bit Error Probability. The end-to-end BER of the relaying schemes described above are given by

$$
\begin{aligned}
P_{\mathrm{SNR}}\{\mathscr{E}\}= & P_{\mathrm{ADF}}\{\mathscr{E}\} P\left\{H_{\mathrm{SNR}}\right\} \\
& +P_{\mathrm{AF}}\{\mathscr{E}\}\left(1-P\left\{H_{\mathrm{SNR}}\right\}\right),
\end{aligned}
$$

for the SNR-based scheme, and by

$$
\begin{aligned}
P_{\mathrm{LLR}}\{\mathscr{E}\}= & P_{\mathrm{ADF}}\{\mathscr{E}\} P\left\{H_{\mathrm{LLR}}\right\} \\
& +P_{\mathrm{AF}}\{\mathscr{E}\}\left(1-P\left\{H_{\mathrm{LLR}}\right\}\right),
\end{aligned}
$$

for the LLR-based scheme. $P_{\mathrm{ADF}}\{\mathscr{E}\}$ and $P_{\mathrm{AF}}\{\mathscr{E}\}$ are the probabilities of error for the ADF and AF relaying protocols, respectively, and $P\left\{H_{\mathrm{SNR}}\right\}$ is the probability that the measured $\Gamma_{S R}$ at $R$ exceeds the threshold $\eta_{\mathrm{SNR}}$, while $P\left\{H_{\mathrm{LLR}}\right\}$ is the probability that the calculated $\Lambda_{S R}$ exceeds the threshold $\eta_{\mathrm{LLR}}$. In $[9,(17)], P_{\mathrm{ADF}}\{\mathscr{E}\}$ is approximated by

$$
P_{\mathrm{ADF}}\{\mathscr{E}\}=\frac{1}{\pi \Omega_{S D} \gamma_{0}^{2} g^{2}}\left(\frac{\alpha}{\Omega_{R D}}+\frac{\beta^{2}}{\pi \Omega_{S R}}\right),
$$

where

$$
\begin{aligned}
& \alpha=\frac{3 \pi(M-1)}{8 M}+\frac{\sin (2 \pi / M)}{4}-\frac{\sin (4 \pi / M)}{32}, \\
& \beta=\frac{\pi(M-1)}{2 M}+\frac{\sin (2 \pi / M)}{4 M}, \\
& g=\sin ^{2}\left(\frac{2 \pi}{M}\right),
\end{aligned}
$$

where $M$ is the modulation order, while $P_{\mathrm{AF}}\{\mathscr{E}\}$ is given in [9, (21)] as

$$
P_{\mathrm{AF}}\{\mathscr{E}\}=\frac{3}{8 \gamma_{0}^{2} g^{2}}\left(\frac{1}{\Omega_{R D}}+\frac{1}{\Omega_{S R}}\right) \frac{1}{\Omega_{S D}} .
$$

$P\left\{H_{\mathrm{SNR}}\right\}$ is given in [13] as

$$
P\left\{H_{\mathrm{SNR}}\right\}=P\left\{\Lambda_{S R}>\eta_{\mathrm{SNR}}\right\}=\operatorname{Exp}\left[-\frac{\eta_{\mathrm{SNR}}}{\sigma_{S R}^{2}}\right],
$$

while $P\left\{H_{\mathrm{LLR}}\right\}$ is given in [13] as

$$
\begin{aligned}
P\left\{H_{\mathrm{LLR}}\right\}= & P\left\{\Lambda_{S R}>\eta_{\mathrm{LLR}}\right\} \\
= & \frac{\operatorname{Exp}\left[-\left(\sqrt{1+\gamma_{S R}^{-1}}+1\right)\left(\eta_{\mathrm{LLR}} / 2\right)\right]}{2\left(\gamma_{S R}+1+\sqrt{\gamma_{S R}^{2}+\gamma_{S R}}\right)} \\
& +\frac{\operatorname{Exp}\left[-\left(\sqrt{1+\gamma_{S R}^{-1}}-1\right)\left(\eta_{\mathrm{LLR}} / 2\right)\right]}{2\left(\gamma_{S R}+1-\sqrt{\gamma_{S R}^{2}+\gamma_{S R}}\right)} .
\end{aligned}
$$


By substituting (10), (12), and (13) in (8), $P_{\text {SNR }}\{\mathscr{E}\}$ is obtained as

$$
\begin{aligned}
P_{\mathrm{SNR}}\{\mathscr{E}\}= & \frac{1}{\pi \Omega_{S D} \gamma_{0}^{2} g^{2}}\left(\frac{\alpha}{\Omega_{R D}}+\frac{\beta^{2}}{\pi \Omega_{S R}}\right) \\
& \cdot \operatorname{Exp}\left[-\frac{\eta_{\mathrm{SNR}}}{\sigma_{S R}^{2}}\right]+\frac{3}{8 \gamma_{0}^{2} g^{2}}\left(\frac{1}{\Omega_{R D}}+\frac{1}{\Omega_{S R}}\right) \\
& \cdot \frac{1}{\Omega_{S D}}\left(1-\operatorname{Exp}\left[-\frac{\eta_{\mathrm{SNR}}}{\sigma_{S R}^{2}}\right]\right) .
\end{aligned}
$$

By substituting (10), (12), and (14) in (9), $P_{\mathrm{LLR}}\{\mathscr{E}\}$ is obtained as

$$
\begin{array}{r}
P_{\mathrm{LLR}}\{\mathscr{E}\}=\frac{1}{\pi \Omega_{S D} \gamma_{0}^{2} g^{2}}\left(\frac{\alpha}{\Omega_{R D}}+\frac{\beta^{2}}{\pi \Omega_{S R}}\right) \\
\cdot\left(\frac{\operatorname{Exp}\left[-\left(\sqrt{1+\gamma_{S R}^{-1}}+1\right)\left(\eta_{\mathrm{LLR}} / 2\right)\right]}{2\left(\gamma_{S R}+1+\sqrt{\gamma_{S R}^{2}+\gamma_{S R}}\right)}\right. \\
\left.+\frac{\operatorname{Exp}\left[-\left(\sqrt{1+\gamma_{S R}^{-1}}-1\right)\left(\eta_{\mathrm{LLR}} / 2\right)\right]}{2\left(\gamma_{S R}+1-\sqrt{\gamma_{S R}^{2}+\gamma_{S R}}\right)}\right) \\
+\frac{3}{8 \gamma_{0}^{2} g^{2}}\left(\frac{1}{\Omega_{R D}}+\frac{1}{\Omega_{S R}}\right) \frac{1}{\Omega_{S D}} \times(1
\end{array}
$$

$$
\begin{gathered}
-\left(\frac{\operatorname{Exp}\left[-\left(\sqrt{1+\gamma_{S R}^{-1}}+1\right)\left(\eta_{\mathrm{LLR}} / 2\right)\right]}{2\left(\gamma_{S R}+1+\sqrt{\gamma_{S R}^{2}+\gamma_{S R}}\right)}\right. \\
\left.\left.+\frac{\operatorname{Exp}\left[-\left(\sqrt{1+\gamma_{S R}^{-1}}-1\right)\left(\eta_{\mathrm{LLR}} / 2\right)\right]}{2\left(\gamma_{S R}+1-\sqrt{\gamma_{S R}^{2}+\gamma_{S R}}\right)}\right)\right) .
\end{gathered}
$$

4.2. Relaying Gain over $A D F$ and $A F$ Protocols. In this subsection, we investigate the performance gains of the proposed hybrid relaying protocol over ADF and AF protocols. Employing the adapted concept of relaying gain $G_{B}^{A}$ (in $\mathrm{dB}$ ), introduced in [9], with $A \in\{$ LLR-based HDAF, SNR-based HDAF $\}$ and $B \in\{\mathrm{ADF}, \mathrm{AF}\}$ and finding the gain in BER performance of the proposed HDAF schemes in comparison to $\mathrm{ADF}$ and $\mathrm{AF}$ protocols, we have

$$
G_{B}^{A}=\frac{10}{2} \times \log _{10}\left[\lim _{\gamma_{0} \rightarrow \infty} \frac{P_{B}\{\mathscr{E}\}}{P_{A}\{\mathscr{E}\}}\right] .
$$

The use of the limit operation in (17) signifies that the computed gain is obtained for the high SNR regime. Substituting (10) and (16) in (17) and carrying out some elementary operations, the gain of the proposed LLR-based HDAF over $\mathrm{ADF}$ protocol can be expressed as

$$
\begin{aligned}
& G_{\mathrm{ADF}}^{\mathrm{LLR}}=5 \log _{10}\left[8 \chi \left(4 \chi\left(\frac{\operatorname{Exp}\left[-(1 / 2)(-1+\xi) \eta_{\mathrm{LLR}}\right]}{1+\gamma_{S R}-\delta}+\frac{\operatorname{Exp}\left[-(1 / 2)(1+\xi) \eta_{\mathrm{LLR}}\right]}{1+\gamma_{S R}+\delta}\right)\right.\right. \\
& \left.\left.\quad+3 \pi \varphi\left(\frac{1-\operatorname{Exp}\left[-(1 / 2)(1+\xi) \eta_{\mathrm{LLR}}\right]}{2\left(1+\gamma_{S R}+\delta\right)}+\frac{\operatorname{Exp}\left[-(1 / 2)(-1+\xi) \eta_{\mathrm{LLR}}\right]}{-2\left(1+\gamma_{S R}-\delta\right)}\right)\right)^{-1}\right]
\end{aligned}
$$

where $\chi=\left(\alpha / \gamma_{R D}+\beta^{2} / \pi \gamma_{S R}\right), \delta=\sqrt{\gamma_{S R}\left(1+\gamma_{S R}\right)}, \varphi=$ $\left(1 / \gamma_{R D}+1 / \gamma_{S R}\right)$, and $\xi=\sqrt{1+1 / \gamma_{S R}}$.
Substituting (12) and (16) in (17) and carrying out some elementary operations, the gain of the proposed LLR-based HDAF over AF protocol can be expressed as

$$
\begin{aligned}
& G_{\mathrm{AF}}^{\mathrm{LLR}}=5 \log _{10}\left[3 \varphi \left(\frac{4}{\pi} \chi\left(\frac{\operatorname{Exp}\left[-(1 / 2)(-1+\xi) \eta_{\mathrm{LLR}}\right]}{1+\gamma_{S R}-\delta}+\frac{\operatorname{Exp}\left[-(1 / 2)(1+\xi) \eta_{\mathrm{LLR}}\right]}{1+\gamma_{S R}+\delta}\right)\right.\right. \\
& \left.\left.\quad+3 \varphi\left(\frac{1-\operatorname{Exp}\left[-(1 / 2)(1+\xi) \eta_{\mathrm{LLR}}\right]}{2\left(1+\gamma_{S R}+\delta\right)}+\frac{\operatorname{Exp}\left[-(1 / 2)(-1+\xi) \eta_{\mathrm{LLR}}\right]}{-2\left(1+\gamma_{S R}-\delta\right)}\right)\right)^{-1}\right]
\end{aligned}
$$

Substituting (10) and (15) in (17) and carrying out some elementary operations, the gain of the proposed SNR-based HDAF over ADF protocol can be expressed as

$$
\begin{aligned}
& G_{\mathrm{ADF}}^{\mathrm{SNR}} \\
& \quad=5 \log _{10}\left[\frac{1}{\operatorname{Exp}\left[-\eta_{\mathrm{SNR}}\right]+3\left(1-\operatorname{Exp}\left[-\eta_{\mathrm{SNR}}\right]\right)}\right] .
\end{aligned}
$$

Finally, substituting (12) and (15) in (17) and carrying out some elementary operations, the gain of the proposed SNRbased HDAF over AF protocol can be expressed as

$$
G_{\mathrm{AF}}^{\mathrm{SNR}}=5 \log _{10}\left[\frac{1}{1-(2 / 3) \operatorname{Exp}\left[-\eta_{\mathrm{SNR}}\right]}\right] .
$$




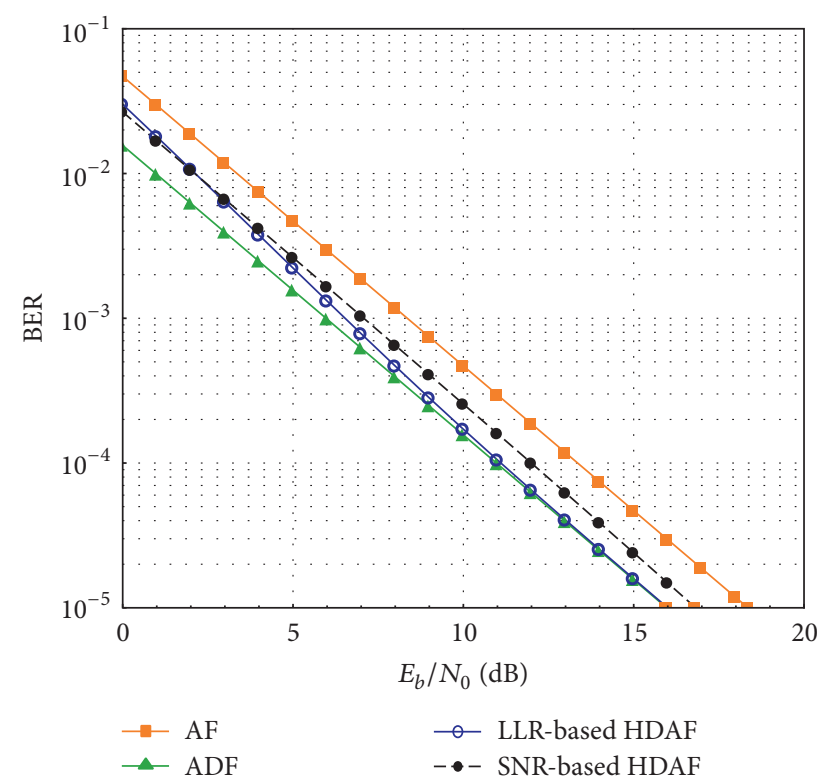

FIGURE 2: BER of the proposed hybrid relaying schemes and AF and ADF protocols for a symmetric cooperative system, where $d_{S R}=$ $d_{R D}=0.5$.

\section{Numerical Results}

In this section, the performance of the proposed relaying scheme is evaluated numerically. The performance is measured in terms of BER curves. It is then compared with different relaying schemes, namely, the hybrid relaying schemes presented in $[6,9,11]$.

Figure 2 shows the BER plotted against the SNR for the proposed LLR- and SNR-based HDAF schemes and AF and ADF protocols. As expected, performance of the proposed schemes is lower-bounded by that of AF protocol and upperbounded by that of ADF protocol. With increasing SNR, the LLR-based scheme shows better error performance than AF protocol, over the SNR-based scheme. This is attributed to the higher sensitivity of the LLR-based scheme over the SNRbased scheme.

BER performance is also plotted against the SNR, for the proposed scheme against other hybrid schemes $[6,9,11]$ for a symmetric relay positioning, with $d_{S R}=d_{R D}=$ 0.5 in Figure 3. The proposed LLR-based HDAF scheme outperforms all the others, including the SNR-based one. For an asymmetric relay positioning, BER performance is plotted against the SNR, in Figures 4 and 5, for $d_{S R}=0.2$ and $d_{S R}=0.8$, respectively. In Figure 4 , as the relay is positioned close to $S$, the proposed LLR-based HDAF scheme outperforms all the other hybrid schemes, followed closely by the SNR-based one. This is because as $d_{S R}$ decreases, the probability of $R$ correctly detecting the signal from $S$ and thus employing ADF protocol increases, which results in an overall improved system performance. In Figure 5, as the relay is positioned close to $D$, only the hybrid scheme of [9] outperforms the proposed LLR- and SNR-based schemes. This is easily justified as follows: since the hybrid scheme of [9] is dependent on the correct detection of the received

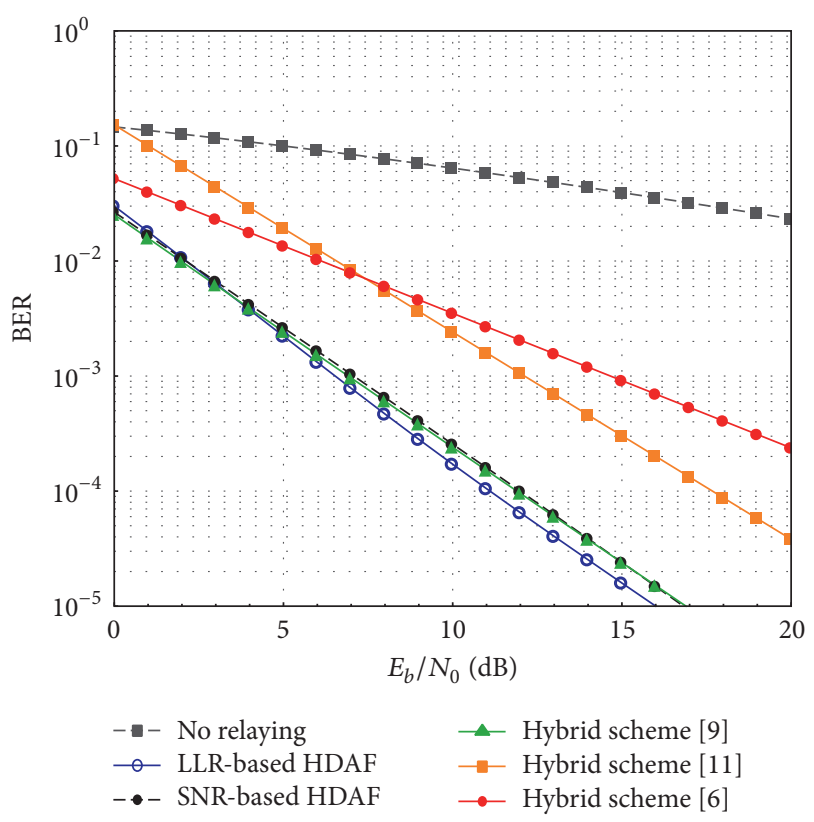

FIGURE 3: BER of the proposed hybrid relaying scheme and various others for a symmetric cooperative system, where $d_{S R}=d_{R D}=0.5$.

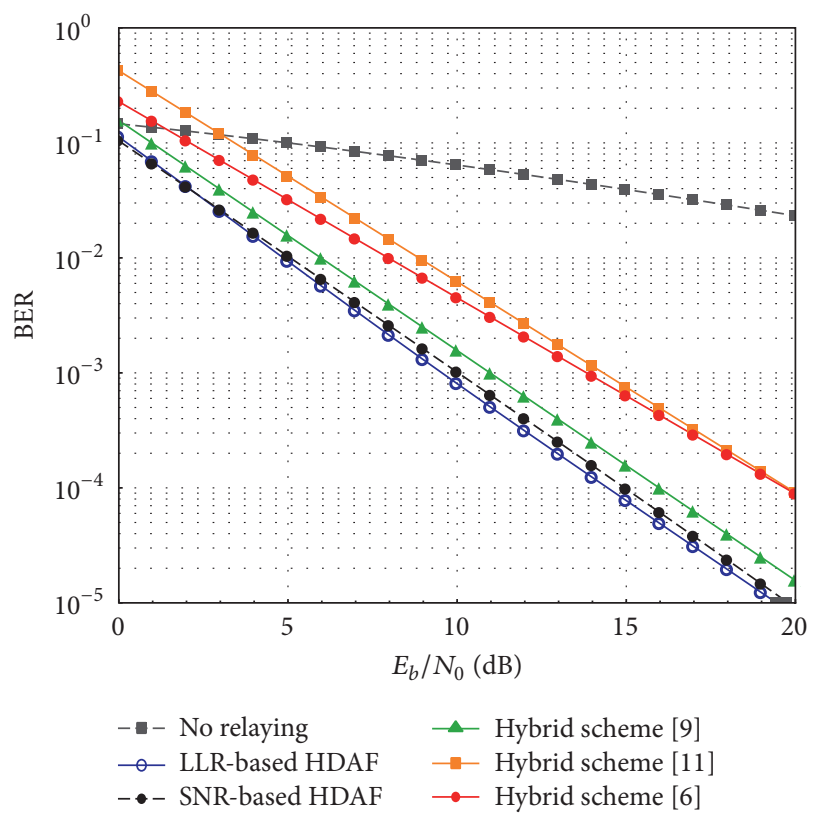

FIGURE 4: BER of the proposed hybrid relaying scheme and various others for an asymmetric cooperative system, where $d_{S R}=0.2$ and $d_{R D}=0.8$.

symbol at $R$, then, as $d_{S R}$ increases, the probability of correct detection decreases and thus $R$ is only active as an AF relay node. This in turn yields better BER performance, since AF protocol always provides better performance as $d_{S R}$ increases.

Tables 1 and 2 give the required SNR values, in $\mathrm{dB}$, to reach a BER of $10^{-3}$ and $10^{-4}$, respectively, by each of the proposed hybrid relaying schemes. It is observed that, in both cases, the LLR-based HDAF performs better than the SNR-based 


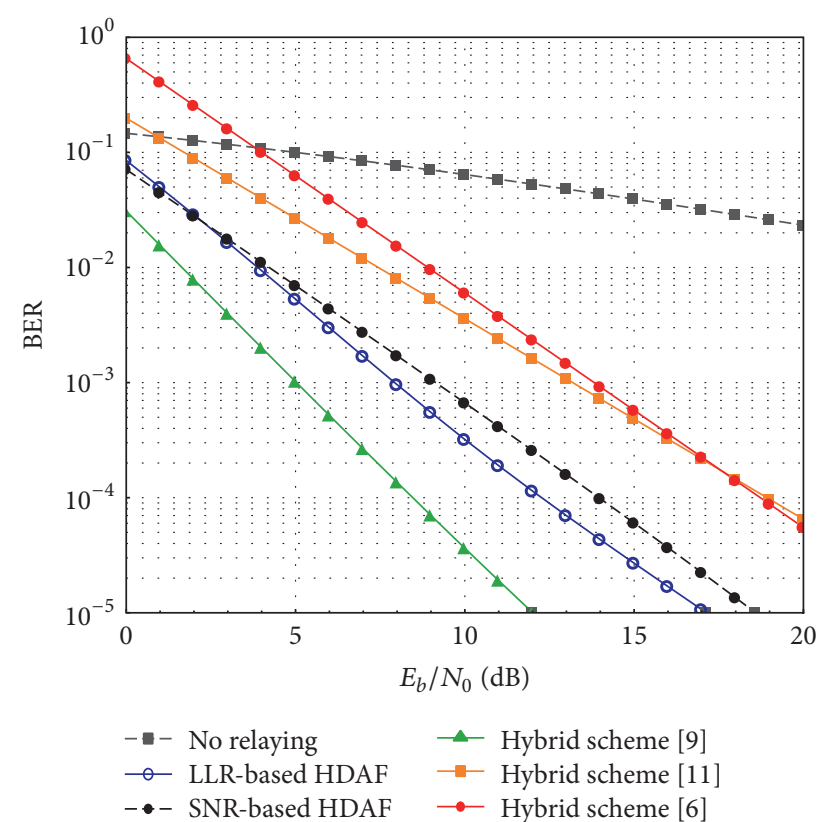

FIGURE 5: BER of the proposed hybrid relaying scheme and various others for an asymmetric cooperative system, where $d_{S R}=0.8$ and $d_{R D}=0.2$.

TABLE 1: A comparison of SNR values at BER of $10^{-3}$ for the proposed hybrid relaying schemes, at various $d_{S R}$.

\begin{tabular}{lccc}
\hline$d_{S R}$ & 0.2 & 0.5 & 0.8 \\
\hline SNR values [dB] for LLR-based HDAF & 9.5 & 6.4 & 7.9 \\
SNR values [dB] for SNR-based HDAF & 10 & 7 & 9 \\
\hline
\end{tabular}

TABLE 2: A comparison of SNR values at BER of $10^{-4}$ for the proposed hybrid relaying schemes, at various $d_{S R}$.

\begin{tabular}{lccc}
\hline$d_{S R}$ & 0.2 & 0.5 & 0.8 \\
\hline SNR values $[\mathrm{dB}]$ for LLR-based HDAF & 14.3 & 11 & 12.1 \\
SNR values $[\mathrm{dB}]$ for SNR-based HDAF & 14.8 & 12 & 13.9 \\
\hline
\end{tabular}

HDAF. Furthermore, it is clear that, for both schemes, the least power is required when $R$ is located at the mid-distance between $S$ and $D$ (i.e., $d_{S R}=0.5$ ).

Finally, Figure 6 shows the gain of several hybrid schemes over AF protocol, namely, the proposed LLR- and SNR-based schemes and that of [9]. It is clear that proposed LLR-based HDAF exhibits increasing gains with increasing SNR and these gains saturate at $\mathrm{SNR} \cong 2.4 \mathrm{~dB}$, irrespective of the value of $\eta_{\text {LLR }}$. It is noted, however, that as the value of $\eta_{\text {LLR }}$ increases (from 0.5 to 2.3) the gain saturates at a slower rate. On the other hand, the proposed SNR-based scheme and the hybrid scheme of [9] introduce constant gains irrespective of the SNR value. Figure 6 also shows that the proposed SNR-based scheme provides gains of $0.15 \mathrm{~dB}$ and $1.1 \mathrm{~dB}$ for $\eta_{\mathrm{SNR}}$ values of 2.3 and 0.5 , respectively, while the hybrid scheme of [9] provides a $1.5 \mathrm{~dB}$ gain over AF protocol.

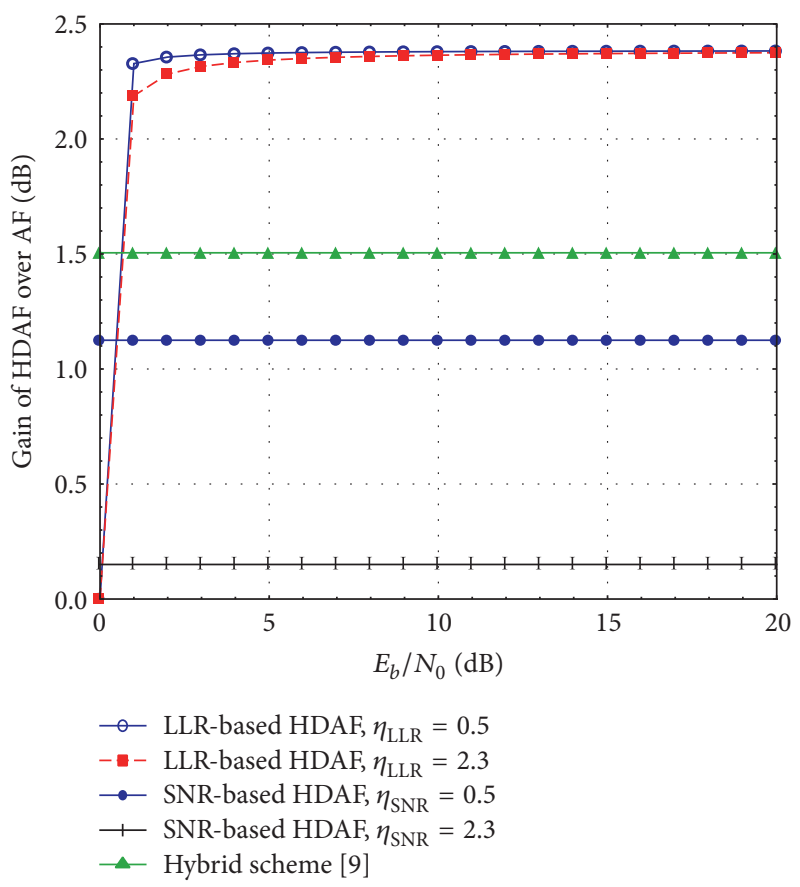

FIGURE 6: Gain of the proposed hybrid relaying schemes and that of the HDAF in [9] over AF protocol for a symmetric cooperative system, where $d_{S R}=0.5$.

\section{Conclusions and Future Works}

In this paper, we derived closed-form expressions for two hybrid relaying schemes for cooperative wireless networks whereby toggling between the ADF and the AF forwarding protocols is based on LLR- and SNR-based thresholds. We have shown that the proposed LLR-based scheme outperforms all existing ones, except for the case of $R$ being very close to $D$, where only the hybrid scheme of [9] proved to be better. The proposed SNR-based hybrid scheme is unlike other SNR-based hybrid schemes found in the literature in that it toggles between ADF and AF protocols, while others toggle between FDF and AF protocols. Finally, the BER gain expressions of both proposed schemes over ADF and AF protocols have been derived.

Future works could include investigating the optimum LLR and SNR thresholds to be used. Also, BER expressions for higher order modulations could be derived for the proposed schemes.

\section{Competing Interests}

The authors declare no competing interests.

\section{References}

[1] T. M. Cover and A. A. El Gamal, "Capacity theorems for the relay channel," IEEE Transactions on Information Theory, vol. 25 , no. 5 , pp. $572-584,1979$.

[2] J. N. Laneman, D. N. C. Tse, and G. W. Wornell, "Cooperative diversity in wireless networks: efficient protocols and outage 
behavior," IEEE Transactions on Information Theory, vol. 50, no. 12, pp. 3062-3080, 2004.

[3] A. Sendonaris, E. Erkip, and B. Aazhang, "User cooperation diversity part I: system description and user cooperative diversity," IEEE Transactions on Information Theory, vol. 51, no. 11, pp. 1927-1938, 2003.

[4] A. Sendonaris, E. Erkip, and B. Aazhang, "User cooperation diversity-part II: implementation aspects and performance analysis," IEEE Transactions on Communications, vol. 51, no. 11, pp. 1939-1948, 2003.

[5] M. O. Hasna and M.-S. Alouini, "End-to-end performance of transmission systems with relays over Rayleigh-fading channels," IEEE Transactions on Wireless Communications, vol. 2, no. 6, pp. 1126-1131, 2003.

[6] W. Alexan and A. El Mahdy, "A hybrid relaying protocol for wireless cooperative networks based on the log-likelihood ratio," in Proceedings of the IEEE Signal Processing: Algorithms, Architectures, Arrangements, and Applications Conference (SPA '15), pp. 128-133, Poznan, Poland, September 2015.

[7] L. Daoud and A. El-Mahdy, "Performance of hybrid relaying in cooperative wireless systems in the presence of impulsive noise," in Proceedings of the IEEE Signal Processing: Algorithms, Architectures, and Applications Conference (SPA '14), pp. 154159, Poznan, Poland, September 2014.

[8] X. Bao and J. Li, "Efficient message relaying for wireless user cooperation: Decode-Amplify-Forward (DAF) and hybrid DAF and coded-cooperation," IEEE Transactions on Wireless Communications, vol. 6, no. 11, pp. 3975-3984, 2007.

[9] T. Q. Duong and H.-J. Zepernick, "On the performance gain of hybrid decode-amplify-forward cooperative communications," EURASIP Journal on Wireless Communications and Networking, vol. 2009, Article ID 479463, 10 pages, 2009.

[10] H. Chen, J. Liu, C. Zhai, and L. Zheng, "Performance analysis of SNR-based hybrid decode-amplify-forward cooperative diversity networks over Rayleigh fading channels," in Proceedings of the IEEE Wireless Communications and Networking Conference (WCNC '10), Sydney, Australia, April 2010.

[11] S. Bouanen, H. Boujemaa, and W. Ajib, "Threshold-based Adaptive Decode-Amplify-Forward relaying protocol for cooperative systems," in Proceedings of the 7th International Wireless Communications and Mobile Computing Conference (IWCMC '11), pp. 725-730, July 2011.

[12] W. Alexan and A. El-Mahdy, "A quantized reliability-relay selection method for wireless cooperative communications," in Proceedings of the 11th International Symposium on Wireless Communications Systems (ISWCS '14), pp. 318-322, IEEE, Barcelona, Spain, August 2014.

[13] H. V. Khuong and H. Y. Kong, "LLR-based decode-and-forward protocol for relay networks and closed-form BER expressions," IEICE Transactions on Fundamentals of Electronics, Communications and Computer Sciences, vol. 89, no. 6, pp. 18321841, 2006. 


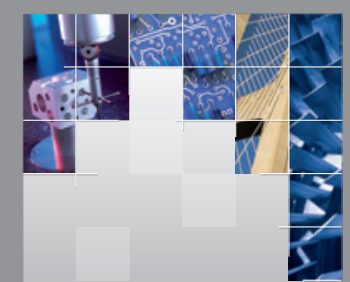

\section{Enfincering}
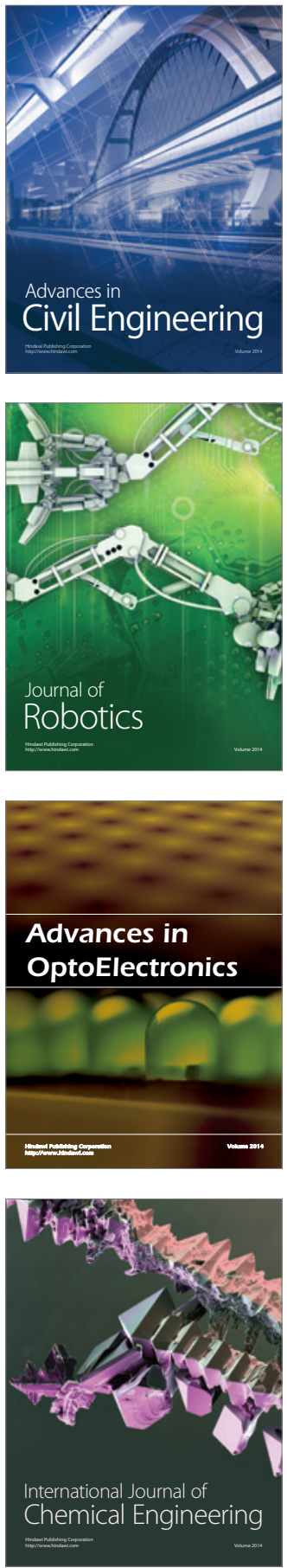

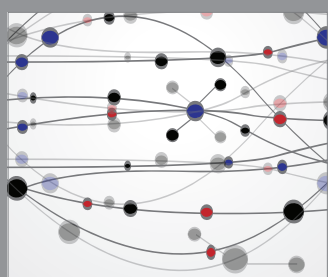

The Scientific World Journal

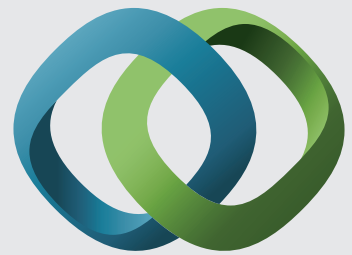

\section{Hindawi}

Submit your manuscripts at

https://www.hindawi.com
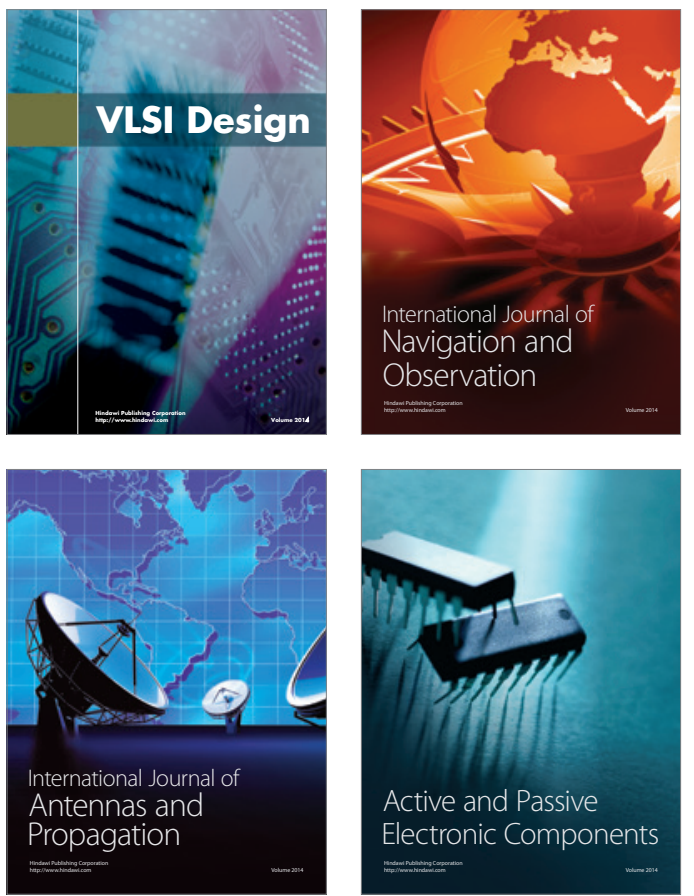
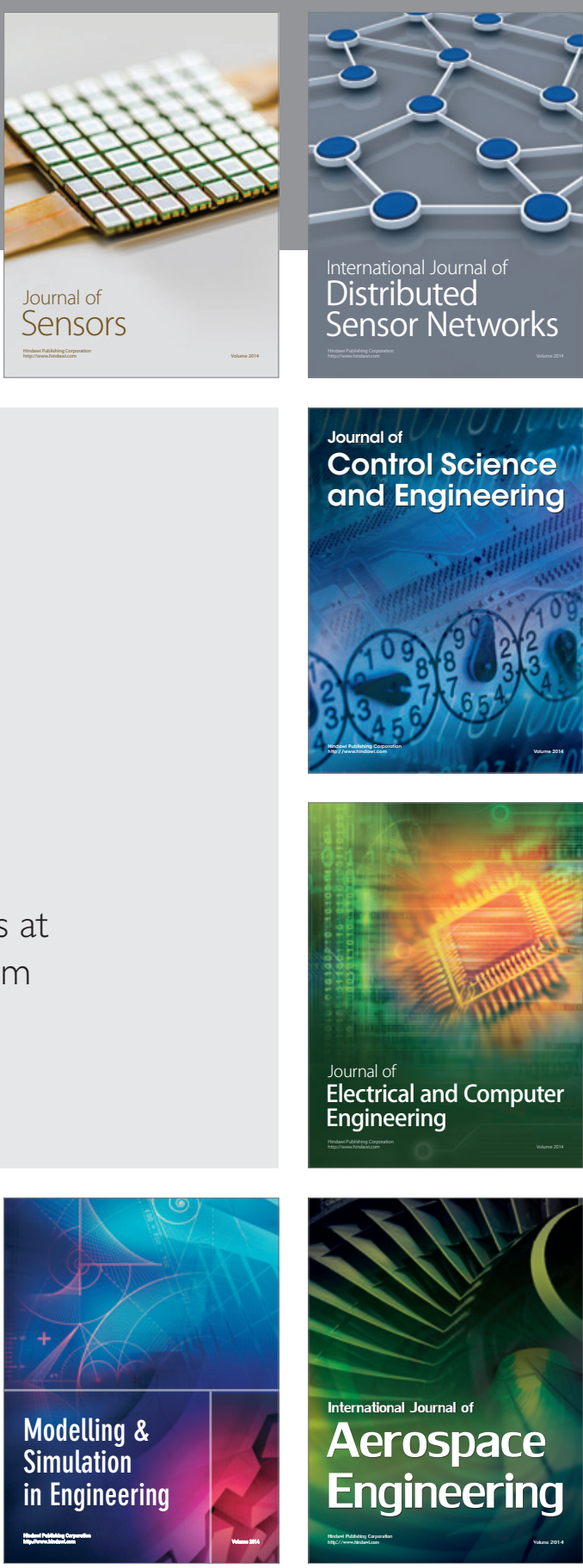

International Journal of

Distributed

Sensor Networks

$-$

Joumal of

Control Science

and Engineering
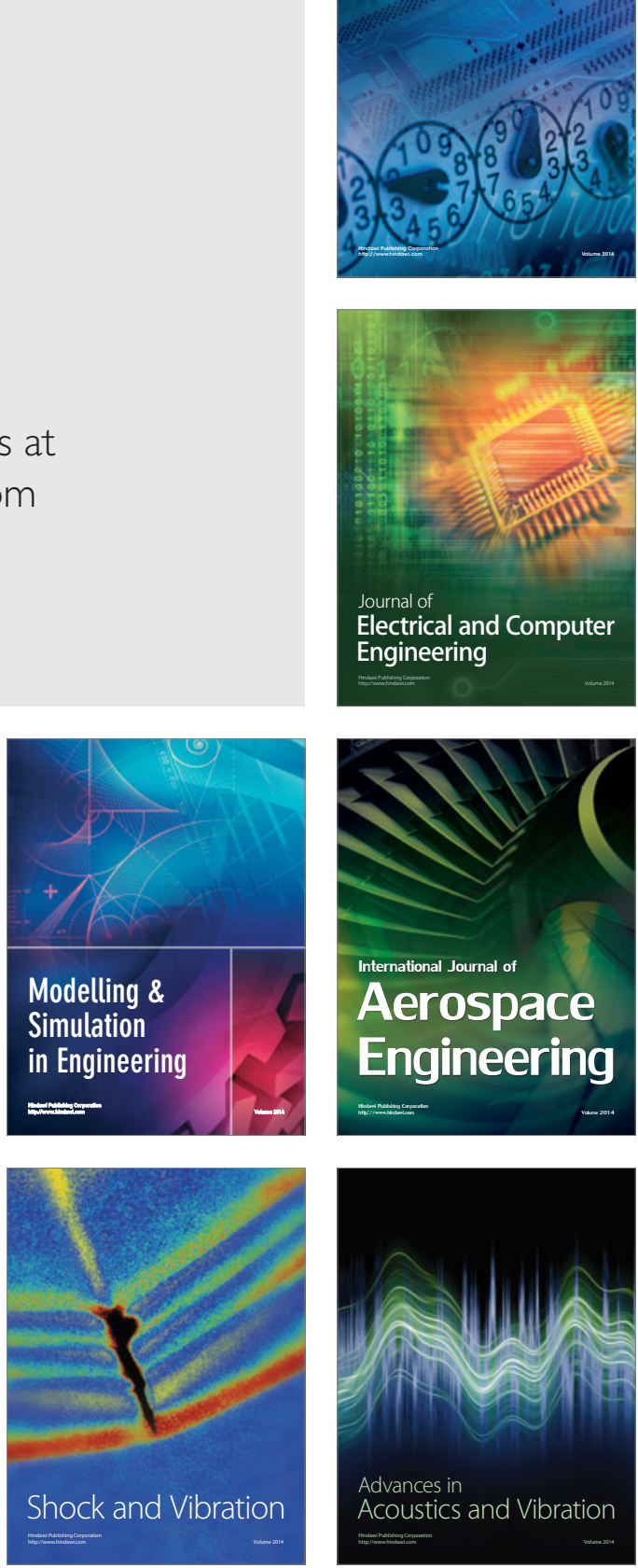